\section{Changes in referral, treatment and outcomes in patients with systemic lupus erythematosus in Germany in the 1990s and the 2000s}

Katinka Albrecht, ${ }^{1}$ Dörte Huscher, ${ }^{1,2}$ Jutta Richter, ${ }^{3}$ Marina Backhaus, ${ }^{2}$ Sascha Bischoff, ${ }^{1}$ Ina Kötter, ${ }^{4}$ Katja Thiele, ${ }^{1}$ Angela Zink, ${ }^{1,2}$ for the German Collaborative Arthritis Centres

To cite: Albrecht K, Huscher D, Richter J, et al. Changes in referral, treatment and outcomes in patients with systemic lupus erythematosus in Germany in the 1990s and the 2000s. Lupus Science \& Medicine 2014;1:e000059. doi:10.1136/lupus-2014000059

Received 16 September 2014 Revised 22 October 2014 Accepted 3 November 2014

\section{(a) CrossMark}

1Epidemiology Unit, German Rheumatism Research Centre, A Leibniz Institute, Berlin, Germany

${ }^{2}$ Department of Rheumatology and Clinical Immunology, Charité University Hospital, Berlin, Germany

${ }^{3}$ Department of Rheumatology, HeinrichHeine-University Duesseldorf, Duesseldorf, Germany

${ }^{4}$ Department of

Rheumatology, Robert-Bosch Hospital, Stuttgart, Germany

Correspondence to Dr Katinka Albrecht; albrecht@drfz.de

\section{ABSTRACT}

Objective: To evaluate trends in the referral, treatment and outcome of patients with systemic lupus erythematosus (SLE) in Germany over two decades. Methods: From 1993 to 2012, 1200 patients with SLE were recorded annually in the national database of the German Collaborative Arthritis Centres. Treatment patterns, healthcare use and outcomes, such as disease activity, function and work participation, were evaluated over time. Furthermore, two distinct cohorts of patients (enrolment 1994-1998, $n=467$; and 20042008, $n=376$ ) observed over 5 years were assessed for changes in outcomes.

Results: The mean disease duration at the first visit to a rheumatologist decreased from 2.6 (1994) to 1.5 (2012) years. Glucocorticoids (69\%), antimalarials $(56 \%)$, azathioprine $(22 \%)$, non-steroidal antiinflammatory drugs $(23 \%)$ and mycophenolate mofetil $(15 \%)$ were the most frequently used treatments in 2012. A significant increase was observed in the use of antimalarials and mycophenolate mofetil. The use of glucocorticoids at $>7.5 \mathrm{mg} /$ day decreased from $27 \%$ (1994) to $10 \%$ (2012). The average length of sick leave taken due to SLE declined from 9 weeks (1997) to 6 weeks (2012). When comparing the two longitudinal cohorts, in the cohort from the 2000s, the intraindividual decline of disease activity was significantly stronger $(p<0.001)$, and fewer patients retired early ( $36 \%$ vs $46 \%$ ).

Conclusions: The disease activity and resource use declined considerably over the observation period, and more patients remained in the labour force. Earlier treatment onset, faster modification of the treatment regimen and more intensive use of anti-inflammatory therapy may account for the improved outcomes in patients with SLE across the years.

\section{INTRODUCTION}

Systemic lupus erythematosus (SLE) is an autoimmune disease of the connective tissue, with extensive variability in the clinical manifestations, course and severity among

\section{KEY MESSAGES}

- Anti-inflammatory treatment of patients with systemic lupus erythematosus has been intensified in the 2000s.

- The disease activity and healthcare use decreased across the years and more patients remained in the labour force.

Patient-reported outcomes are still to be improved.

patients, as well as in individual patients over time. In the past decades, the mortality rates have decreased considerably, and SLE as a chronic disease now has a 5-year survival rate of $>90 \% .{ }^{12}$ Treatment with immunosuppressive drugs can help to keep organ involvement manageable and allow many patients to achieve remission or at least maintain a low disease activity. ${ }^{3}$

Observational studies have demonstrated a decreasing disease activity during follow-up within SLE cohorts. ${ }^{4-6}$ A trend across calendar years was also seen by Urowitz et al when comparing the mean SLE disease activity index (SLEDAI) between four cohorts with study entries between 1970 and $2005^{4-7}$ Disease activity is predominantly defined by disease activity indices and/or physician assessments. ${ }^{6}$ However, cross-sectional data demonstrated that patient assessment of SLE disease activity is discordant, which might be explained by a difference in the focus on the physical (physician assessment) or psychological (patient assessment) effects of the disease. ${ }^{8-10}$ Data on the development of other outcomes, such as pain, healthcare use and work participation, during the course of SLE are limited. ${ }^{11-13}$ With regards to treatment patterns, it remains difficult to differentiate between changes in therapies that are prompted by the clinical 
status of an individual patient and changes in treatment practices over time. We examined cross-sectional data from patients with SLE who were enrolled in an annual patient register between 1993 and 2012. In a subsidiary analysis, longitudinal data from patients enrolled either in the 1990s or in the 2000s were evaluated with regards to individual treatment modifications, clinical and patient-reported outcomes and changes in work participation.

\section{PATIENTS AND METHODS}

The national database of the German Collaborative Arthritis Centres is an ongoing prospective study that was established in 1993 as a long-term monitoring system for German rheumatology patients. ${ }^{14}$ The database contains annually updated clinical data and patientreported outcomes for unselected outpatients with inflammatory rheumatic diseases. Patients with a confirmed diagnosis of SLE were eligible for the analysis. Cross-sectional data for each of the years from 1993 to 2012 were compared with regards to drug treatment, physician and patient-reported assessments, healthcare use and work participation. The clinical dataset included demographic data, disease duration, medical treatment, disease activity (physician global assessment on a 0-10 numerical rating scale (NRS), 0 reflecting no disease activity and 10 reflecting very high disease activity) and disease severity (qualitative assessment by the physician on a five-item Likert scale (asymptomatic, mild, moderate, severe or very severe). Patients reported their global health status (NRS $0-10$ as of 2000), pain (NRS 0-10) and functional status. The latter was assessed by the Hannover Functional Status Questionnaire $(\mathrm{FFbH})$, indicating the percentage of full-functional capability ranging from 0 to 100 . This instrument is widely used in Germany and is comparable to the Health Assessment Questionnaire (HAQ). Both instruments are highly correlated, and $\mathrm{FFbH}$ values can be transformed into $\mathrm{HAQ}$ values. ${ }^{15}$ We report the HAQ values in this paper. Work participation was evaluated as employment and early retirement rates for patients aged $<65$ years. The frequency and duration of sick leave were analysed for employed patients. Hospital stays and the mean number of days of hospitalisation were used as indicators of resource use. Due to a changeover from paper-based to IT-based data collection accompanied by transition difficulties in the participating units, the data from 2005 and 2006 were not sufficiently complete for inclusion in this study.

A subanalysis was conducted for patients with incident disease (<1-year disease duration) regarding the symptom duration at the first referral to rheumatologic care, disease activity and initial treatment.

A second subanalysis on longitudinal developments related to treatment, outcomes and work participation was compared in two distinct cohorts: patients enrolled in 1994-1998 (cohort 1) and patients enrolled in 20042008 (cohort 2), who were followed annually for at least 4 years. One missing documentation within this period was accepted. Both cohorts were tested for intracohort time trends. Differences between the two decades were compared based on the measurements at the end of the observation period.

\section{Statistical analysis}

To test for homogeneous time trends, simple trend tests based on the mean square successive differences were performed. ${ }^{16}$ For comparison of frequencies, $\chi^{2}$ test or Fisher's exact test was used, and t test or Mann-Whitney test was used for continuous variables. No adjustment for multiple testing was performed. The data were analysed using IBM SPSS Statistics, V.19.

\section{RESULTS}

\section{Cross-sectional data}

Patient characteristics

Between 1993 and 2012, the numbers of patients with SLE eligible for the analysis ranged between 850 and 1300 annually (table 1 ). The patient data were documented in tertiary care centres $(87 \%)$ or in private practices $(13 \%)$. In total, $89 \%$ of the patients were women. The mean age was 42 years in 1993 and increased to 45 years in 2012. The mean disease duration was 11 years in 1993 with an increase of 4 years during the observed period.

Table 1 Demographic and disease-related characteristics of patients with systemic lupus erythematosus in the national database of the German Collaborative Arthritis Centres

\begin{tabular}{lrrrrrrrr}
\hline & $1993^{*}$ & $\mathbf{1 9 9 6}$ & $\mathbf{1 9 9 9}$ & $\mathbf{2 0 0 2}$ & $\mathbf{2 0 0 4}$ & $\mathbf{2 0 0 7}$ & $\mathbf{2 0 1 0}$ & $\mathbf{2 0 1 2}$ \\
\hline $\mathrm{N}$ & 1249 & 1359 & 1701 & 1369 & 1040 & 846 & 986 & 914 \\
Sex, female (\%) & 90 & 90 & 89 & 88 & 89 & 89 & 89 & 90 \\
Mean age, years & 42.4 & 42.8 & 44.2 & 45.0 & 46.1 & 45.8 & 45.6 & 46.4 \\
Mean disease duration, years & 9.2 & 9.0 & 9.4 & 10.1 & 10.8 & 11.9 & 13.1 & 14.0 \\
Mean age at disease onset, years. & 31.2 & 32.0 & 33.0 & 32.6 & 33.0 & 32.2 & 31.0 & 30.7 \\
Disease severity†, (\%) asymptomatic/mild & 46.4 & 45.3 & 49.4 & 43.5 & 40.7 & 44.7 & 45.5 & 46.1 \\
Moderate & 38.4 & 38.6 & 36.4 & 41.6 & 41.5 & 44.6 & 43.1 & 41.8 \\
(Very) Severe & 15.2 & 16.1 & 14.1 & 14.9 & 17.8 & 10.7 & 11.4 & 12.1 \\
\hline
\end{tabular}

${ }^{*}$ For better readability, only data of every second to third year is shown.

†The disease severity was assessed by the physician. 
A total of $45 \%$ of the patients were characterised as having mild disease, $41 \%$ had moderate disease and $14 \%$ had severe or very severe disease in 1993 with a decline of $3 \%$ in the latter group during the observed period.

\section{First referral to rheumatologic care}

Between 1993 and 2008, the percentage of patients consulting a rheumatologist within 6 months after symptom onset increased from 63 to $76(\mathrm{p}<0.01)$ (available case numbers thereafter were $<50$ per year and considered too low for evaluation). The mean disease duration at the first rheumatologic visit decreased from 2.6 (1994) to 1.5 years $(2012)$.

\section{Drug treatment}

Figure 1A shows the use of anti-inflammatory treatment between 1993 and 2012. The use of antimalarials increased from $36 \%$ to $56 \%$ in 2012. The use of azathioprine (38-22\%) and cyclosporine A (6-3\%) decreased across the years. Mycophenolate mofetil has been prescribed since 2005, and its prescription rate increased to $15 \%$ in 2012. Methotrexate was used in $\sim 7 \%$ of patients and cyclophosphamide in $\sim 3 \%$ of patients across the years. A total of $4 \%$ of patients received biologics in 2012. The number of patients treated with glucocorticoids $>7.5 \mathrm{mg} /$ day decreased from $27 \%$ in 1996 to $10 \%$ in 2012 ( $p<0.001)$, whereas glucocorticoids $\leq 7.5 \mathrm{mg} /$ day were more frequent in 2012. Pain medication with analgesics increased from $6 \%$ in 1993 to $20 \%$ in $2012(\mathrm{p}<0.001)$.

\section{Clinical outcomes}

Between 1993 and 2012, the percentage of patients with low disease activity assessed by the physicians increased from 69 to 88 (figure 1B). The mean patient assessment
Figure 1 Changing patterns in patients with systemic lupus erythematosus (SLE) between 1993 and 2012. NRS, numerical rating scale; NSAID, non-steroidal anti-inflammatory drugs.
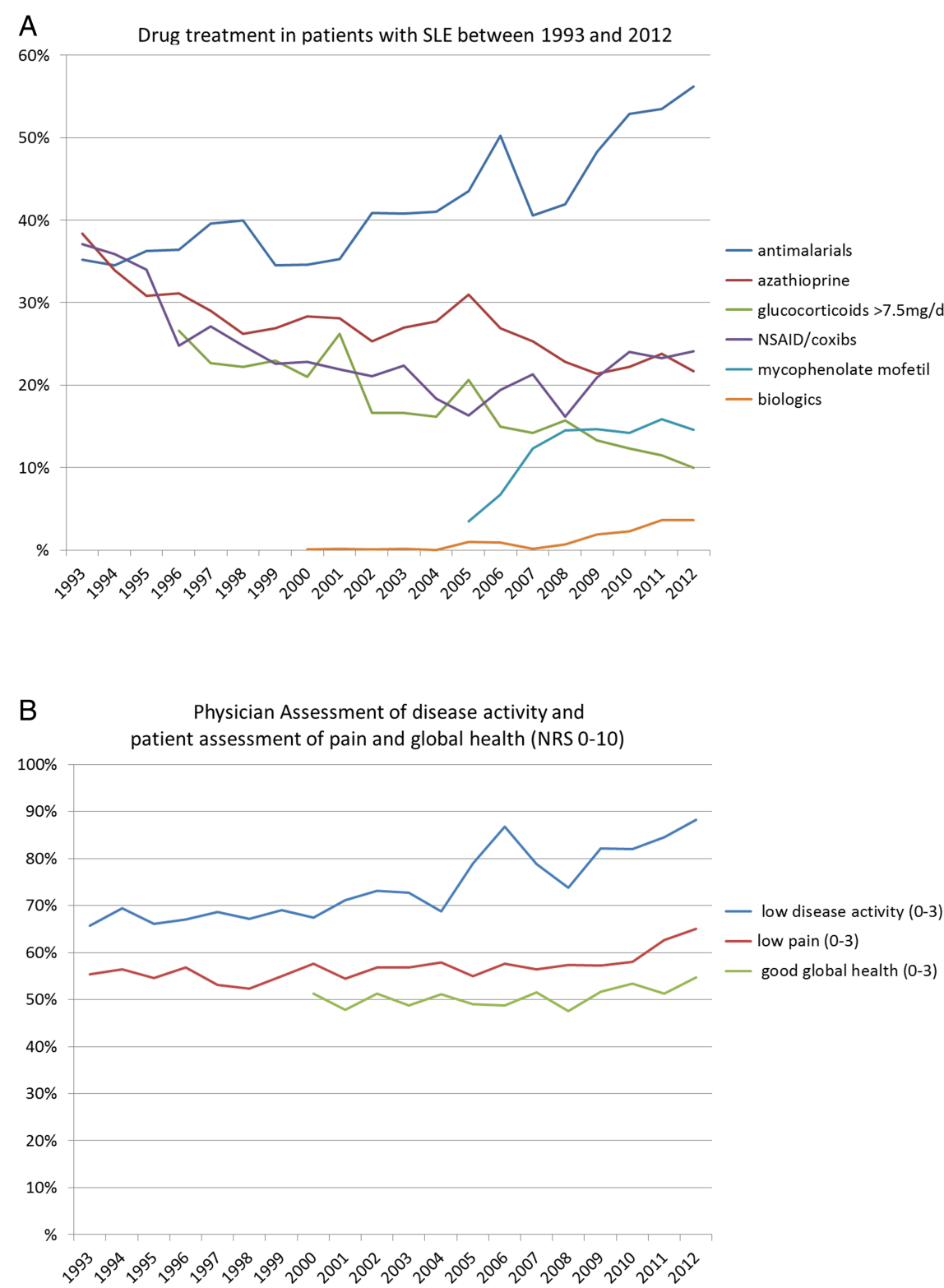
of pain on a NRS $0-10$ decreased from 3.3 to 2.8 $(\mathrm{p}<0.001)$, and the percentage of patients with severe pain $(7-10)$ decreased from 17 to $13(\mathrm{p}<0.001)$. The mean patient-reported global health on an NRS 0-10 (assessed since 2000) remained unchanged (3.7), while the percentage of patients with poor global health decreased. There was a $10 \%$ increase in the number of patients with good function $(\mathrm{HAQ} \leq 0.5)$ while the percentage of patients with severely affected function $(\mathrm{HAQ}>2)$ remained at $6 \%$.

\section{Healthcare use and work participation}

The percentage of patients who had been hospitalised during the preceding year showed a continuous decline from 27 in 1993 to 14 in 2012. The average length of stay declined from 32 days in 1993 to 17 days in 2012. The average length of sick leave due to SLE declined from 9 weeks in 1997 to 6 weeks in 2012. The percentage of employed patients $<65$ years increased continuously from 45 in 1993 to 51 in 2012, except for patients $<40$ years. The greatest increase $(\Delta+12 \%)$ was found in patients aged $41-50$ years. The percentage of patients in early retirement $(\sim 24 \%)$ did not change significantly across the years.

\section{Patients with incident SLE}

To evaluate whether the clinical presentation at the onset of treatment has changed over time, we compared patients with incident SLE (disease duration at first visit $<1$ year) in the 1990s $(n=567)$ and in the 2000s $(n=467)$. Patients did not differ significantly concerning sex, age, disease duration, mean $\mathrm{FFbH}$ values and erythrocyte sedimentation rate. The proportions of patients with a severe disease or high activity were non-significantly lower in the 2000s. Anti-inflammatory therapy was comparable except for a decline in the use of glucocorticoids and a more frequent use of non-steroidal anti-inflammatory drugs (NSAIDs). The percentage of employed patients <65 years was lower in the 2000s (55\%) compared with the 1990s $(61 \%)$.

\section{Longitudinal data of patients with prevalent SLE and a 4-year follow-up}

Patient characteristics of the two cohorts

A total of 467 patients with a follow-up of 4 years enrolled in 1994-1998 (cohort 1) and 376 patients enrolled in 2004-2008 (cohort 2) were evaluated (table 2). At the first documentation year, the median disease duration was 1.2 years shorter, and the percentage of patients with asymptomatic or low disease activity was non-significantly higher in cohort $2(\mathrm{p}=0.076)$.

\section{Drug treatment}

In both cohorts, glucocorticoids, antimalarials, NSAIDs and azathioprine were the most frequently used substances at baseline (figure 2A). The use of immunosuppressive drugs remained stable in both cohorts, but antimalarials were used increasingly over time in cohort
Table 2 Demographic and disease-related characteristics of patients with systemic lupus erythematosus enrolled in 1994-1998 (cohort 1) or in 2004-2008 (cohort 2)

\begin{tabular}{lll}
\hline & Cohort 1 & Cohort 2 \\
\hline Inclusion in the registry & $1994-1998$ & $2004-2008$ \\
$\mathrm{~N}$ & 467 & 376 \\
Sex, female (\%) & 88 & 87 \\
Mean age at first & $41.3 \pm 13.9$ & $42.6 \pm 14.6$ \\
documentation, years $( \pm \mathrm{SD})$ & & \\
Disease duration $\leq 5$ years at & $203(44)$ & $138(37)$ \\
first documentation, $\mathrm{n}(\%)$ & & \\
Disease activity at first documentation*, NRS & $0-10(\%)$ \\
$\quad$ Low (0-3) & 65 & 72 \\
$\quad$ Moderate (4-6) & 27 & 25 \\
$\quad$ High (7-10) & 9 & 3 \\
Disease severity at first documentation & $(\%)$ \\
$\quad$ Asymptomatic/low & 47 & 40 \\
$\quad$ Moderate & 41 & 48 \\
$\quad$ Severe & 12 & 12 \\
\hline
\end{tabular}

${ }^{*}$ The disease activity and severity was assessed by the physician. NRS, numerical rating scale.

$2(\mathrm{p}<0.001)$. When comparing both cohorts, in cohort 2 , treatment changes were performed more frequently and the use of combinations of immunosuppressive substances with antimalarials more than doubled $(p<0.001)$ (data not shown). The use of glucocorticoids $>7.5 \mathrm{mg} /$ day decreased in both cohorts and was significantly lower at the last observation in cohort 2 compared with cohort $1 \quad(\mathrm{p}<0.05)$. During the observation period, the use of NSAIDs and analgesics increased considerably in cohort 2 and was significantly higher in comparison to cohort $1 \quad(p<0.001)$. Prophylaxis or treatment of osteoporosis with calcium and vitamin $\mathrm{D}$ and/or bisphosphonates was used three times more frequently at the first documentation year in cohort $2(21 \%$ vs $60 \%)$.

\section{Clinical outcomes}

Figure 2B shows the physician's assessments of disease activity and the patient's assessment of pain and global health. The increase of the percentage of patients with low disease activity (0-3) during follow-up was greater in cohort 2, and the difference between the cohorts at year 4 was significant $(\mathrm{p}<0.001)$. The percentage of patients reporting severe pain decreased in cohort 2 from 16 to $12(\mathrm{p}<0.05)$. The longitudinal trend in the patient's assessments of global health and pain is discordant with the significant decrease in disease activity as assessed by the physicians $(p<0.01)$. The percentage of patients with good functional status $(\mathrm{HAQ} \leq 1)$ remained unchanged in cohort 1 during follow-up (69\% to 68\%) and increased in cohort 2 ( $72 \%$ to $77 \%)$.

\section{Healthcare use and work participation}

In cohort $1,55 \%$ of patients $<65$ years were employed at the first documentation. Four years later, 39\% were still employed. In cohort 2, though starting with a similar employment rate $(57 \%)$, the rate of $48 \%$ at year 4 was 
Figure 2 Treatment and clinical outcomes during a 4-year follow-up in the 1990s (cohort 1) and in the 2000s (cohort 2). NRS, numerical rating scale; NSAID, non-steroidal anti-inflammatory drugs.
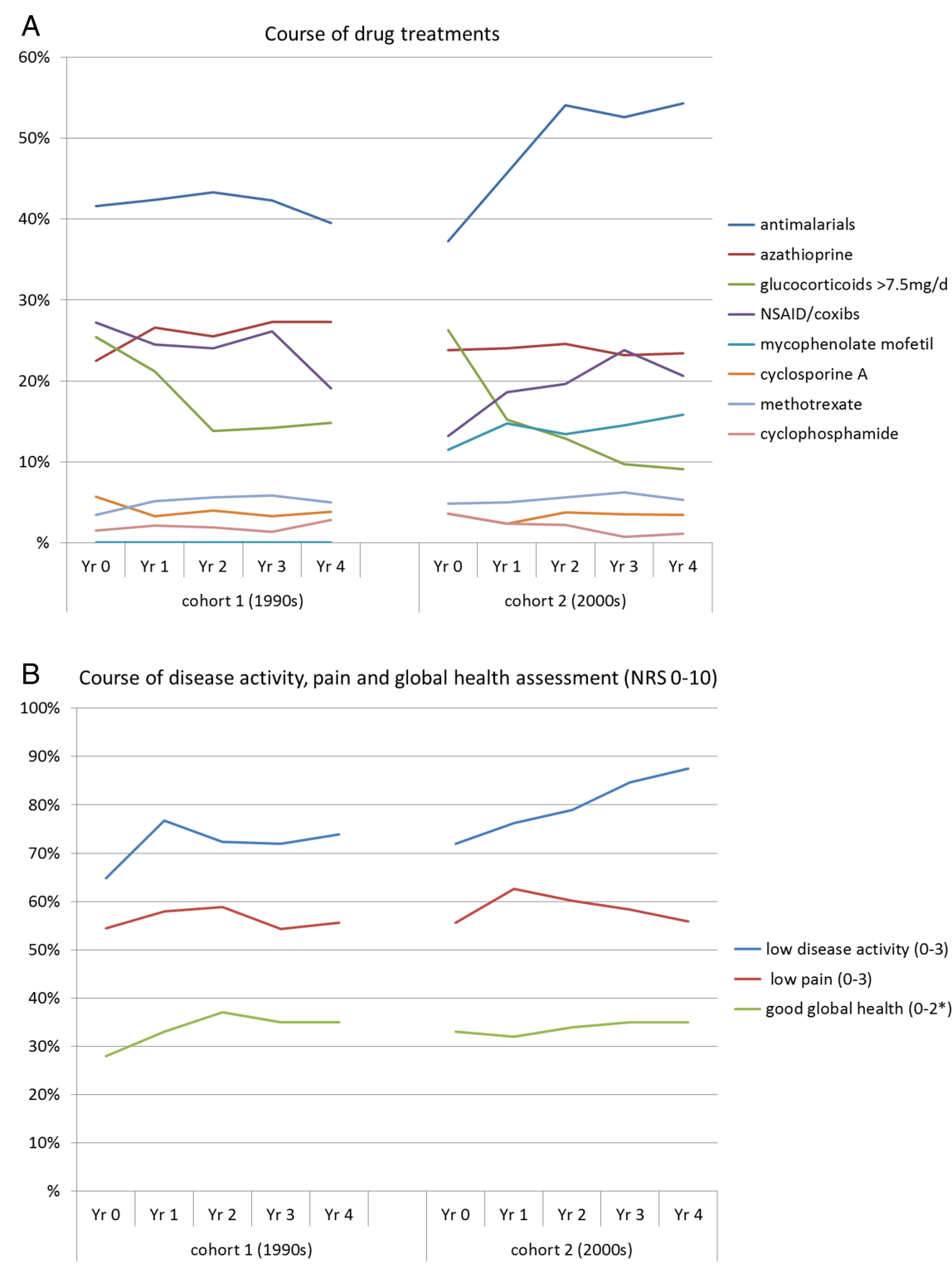

considerably higher compared with cohort 1 , particularly in patients with a disease duration $>5$ years (data not shown). In cohort 2 , fewer patients $<65$ years retired early during the observation period ( $36 \%$ vs $46 \%$ ). In both cohorts, the frequencies of hospitalisation and sick leave declined significantly during the observation period $(p<0.05)$. The mean number of days of sick leave (20 vs 41 days per year) and the mean number of days in the hospital (11 vs 31 days per year) were remarkably lower in cohort 2 when comparing both cohorts at the end of the observation.

\section{DISCUSSION}

The management of rheumatic diseases has improved considerably in the past decade. While the number of therapeutic options in rheumatoid arthritis (RA) and spondyloarthritides has grown tremendously with the entry of biologic therapies, SLE is just beginning to become a focus of these treatment regimens. ${ }^{17}$ On this account, we evaluated whether the use of treatment options that have been available for a long time has changed and whether clinical and patient-reported outcomes as well as healthcare use and participation in the labour market improved in the 2000s compared with the previous decade.

Our cross-sectional data show an increasing use of moderate anti-inflammatory therapy such as antimalarials, low-dose glucocorticoids and NSAIDs. The current treat-to-target recommendations in SLE advise us to give serious consideration to the use of antimalarials irrespective of the use of other treatments. ${ }^{18}$ With this in mind, the increase in the percentage of patients on antimalarials to about $50 \%$ is encouraging but not sufficient yet. The regular use of antimalarials will assumingly become more widespread in the coming years. Additionally, the decrease in the proportion of patients on high-dose glucocorticoids over time is a step in the right direction, yet without reaching the recommendation to "aim for the lowest glucocorticoid dosage needed 
to control disease or to withdraw glucocorticoids completely as possible". ${ }^{18}$

The disease activity as assessed by the physician decreased across the years, and we saw a considerable decline in healthcare use. As our annually updated data comprise consecutive patients in routine outpatient care, we cannot exclude variations in patient selection. Therefore, the increasing mean disease duration over the years could be caused by a difference in patient selection or reflect an older-growing SLE population in rheumatological care. The clinical improvements could be explained by a decline in SLE activity with ongoing disease. To control for this possible bias, we performed a subanalysis in patients with incident SLE in our crosssectional data. In addition, we analysed patients who were seen in at least five subsequent years, stratified by disease duration. Our results support various explanations for the improved outcomes. First, in the more recent years, patients were referred earlier to the rheumatologist. During the time period, the number of rheumatologists increased in Germany, leading to better access to care.(Kopetsch T. Data from the Federal Register of Physicians. Federal Association of Statutory Health Insurance Physicians, personal communication, 2013). Consequently, patients might have less severe manifestations at the time of referral. However, the analysis of incident cases shows that the disease severity and activity at the first visit to a rheumatologist were only marginally better than in the 1990s, and the employment rate was even lower. Second, the follow-up of patients with prevalent SLE shows that in the 2000s drug treatment was adapted more frequently and the use of combination therapy with anti-inflammatory substances increased. Additionally, complementary therapies, such as pain medication and osteo-protective therapy, were used more frequently. When comparing the two cohorts, we observed a stronger decline of disease activity and resource use during the 4-year follow-up as well as a better maintained functional status in the 2000s cohort. Moreover, although the employment rates were similar at the initial visit, more patients remained in the work force in the 2000s cohort, especially patients with a disease duration of $>5$ years. This is of note as a recent cohort study reported a $50 \%$ work loss among patients with SLE with an average disease duration of 13 years. ${ }^{19}$ A higher risk of work loss was seen among patients aged $55-64^{13}$ and with high disease activity and organ manifestation. ${ }^{13}{ }^{19}$ In the literature, a time trend towards lower rates of work disability in patients with SLE is only reported from cross-sectional data in comparison to previous reports. ${ }^{12}$ In our data, the sick leave and hospitalisation rates decreased substantially across the years. A current analysis of social insurance data in Germany confirms that, over the last decade, the average decline in sick leave and in the retirement rates of patients with SLE was higher compared with all non-inflammatory diseases. ${ }^{20}$ During the observed time period, the length of stay in hospital has generally decreased in Germany. ${ }^{21}$
However, it is encouraging that we saw a decline similar to other diagnoses such as $\mathrm{RA}^{22}$ in the challenging subset of patients with SLE. The decline in organ manifestations confirms the decline of disease activity in the 2000s and is consistent with the declining disease activity in other cohorts. ${ }^{4-6}$ At least in part this result can be explained by the use of more effective treatment strategies. ${ }^{3}$ In an earlier analysis of data from our national database, we showed that with increasing disease duration, the deterioration in patient-reported outcomes was much lower in SLE compared with patients with RA. ${ }^{23}$ In accordance with reported analyses of other cohorts, global health and pain assessments do not conform to physicians' assessments of disease activity. ${ }^{9} 1024$ Our data also show that a good control of disease activity in the course of SLE, as observed by the physician, does not necessarily reflect a similar improvement in patientreported outcomes. Data from the German Lupus Erythematosus Self-Help Organisation cohort confirm ongoing moderate pain and impairment levels. ${ }^{11} \mathrm{We}$ showed that there is an increasing gap between physician ratings and patient-reported outcomes in other inflammatory rheumatic diseases as well. ${ }^{25}$ Thus, an important objective in SLE and other rheumatic diseases will be to focus on the improvement of health-related quality of life that is addressed as an independent target in the current recommendations. ${ }^{18}$

The main limitations of our study are that SLE-specific indices regarding disease activity and organ damage, such as SLEDAI, System Lupus International Collaborating Clinics or European Consensus Lupus Activity Measurements (ECLAM), were not available for the entire period of observation. ECLAM was introduced in the national database in 2007 when it became an increasing part of routine rheumatologic care. Because the national database has been a routine monitoring instrument for all inflammatory rheumatic diseases during the last 20 years, for the years 1993-2006, only generic instruments regarding physician and patient global assessments were available. However, we are convinced that these generic instruments are able to reflect global changes in the outcomes of SLE.

In summary, our data show considerable declines in disease activity and healthcare use as well as an increase in work participation in the 2000s. We suggest that in addition to earlier referral to rheumatologic care, a faster treatment modification with an intensive use of moderate anti-inflammatory therapy is the main reason for the better outcomes in patients with SLE across the years. Nevertheless, patient-reported outcomes are still to be improved.

Acknowledgements The authors gratefully acknowledge the contributions and the enthusiasm of all participating consultant rheumatologists who contributed data of their patients with inflammatory rheumatic diseases to the National Database since 1993. In particular, the authors would like to acknowledge the significant contributions of R Alten (Berlin), M Aringer (Dresden), H Burkhardt (Frankfurt/Main), R de la Camp (Erlangen), T Eidner (Jena), K Fischer (Greifswald), J Henes (Tübingen), U von Hinüber 
(Hildesheim), G Hoese (Stadthagen), K Karberg (Berlin), A Krause (Berlin), W Ochs (Bayreuth), S Späthling-Mestekemper (München) and S Wassenberg (Ratingen).

Contributors All authors were involved in drafting the article or revising it critically for important intellectual content, and all authors approved the final version to be published. DH had full access to all of the data in the study and takes responsibility for the integrity of the data and the accuracy of the data analysis. Study conception and design: $\mathrm{KA}, \mathrm{DHr}$ and $\mathrm{AZ}$. Acquisition of data: $\mathrm{MB}, \mathrm{SB}, \mathrm{IK}, \mathrm{JR}$ and $\mathrm{KT}$. Analysis and interpretation of data: $\mathrm{KA}, \mathrm{DH}, \mathrm{JR}, \mathrm{MB}$, $\mathrm{IK}$ and $\mathrm{AZ}$.

Funding The National Database was funded by the Federal Minister of Research from 1999 to 2007 [01GI0344/3]. Since 2007, the database has been funded by unconditional grants from the German Collaborative Arthritis Centres and from a consortium of 11 pharmaceutical companies to the German Academy for Continuing Medical Education in Rheumatology.

Competing interests None.

Patient consent Obtained.

Ethics approval Ethics committee of the Charité-University Medicine Berlin (EA1/196/06).

Provenance and peer review Not commissioned; externally peer reviewed.

Data sharing statement No additional data are available.

Open Access This is an Open Access article distributed in accordance with the Creative Commons Attribution Non Commercial (CC BY-NC 4.0) license, which permits others to distribute, remix, adapt, build upon this work noncommercially, and license their derivative works on different terms, provided the original work is properly cited and the use is non-commercial. See: http:// creativecommons.org/licenses/by-nc/4.0/

\section{REFERENCES}

1. Ruiz E, Ramalle-Gomara E, Elena A, et al. Trends in systemic lupus erythematosus mortality in Spain from 1981 to 2010. Lupus 2014;23:431-5

2. Shen HN, Yang HH, Lu CL. Temporal trends in characteristics and outcome of intensive care unit patients with systemic lupus erythematosus in Taiwan: a national population-based study. Lupus 2013;22:644-52.

3. Cervera R, Khamashta MA, Hughes GR. The Euro-lupus project: epidemiology of systemic lupus erythematosus in Europe. Lupus 2009:18:869-74.

4. Urowitz MB, Gladman DD, Ibanez D, et al. Evolution of disease burden over five years in a multicenter inception systemic lupus erythematosus cohort. Arthritis Care Res (Hoboken) 2012;64:132-7.

5. Nossent J, Kiss E, Rozman B, et al. Disease activity and damage accrual during the early disease course in a multinational inception cohort of patients with systemic lupus erythematosus. Lupus 2010;19:949-56.

6. Zen M, Bassi N, Nalotto L, et al. Disease activity patterns in a monocentric cohort of SLE patients: a seven-year follow-up study. Clin Exp Rheumatol 2012;30:856-63.

7. Urowitz MB, Gladman DD, Tom BD, et al. Changing patterns in mortality and disease outcomes for patients with systemic lupus erythematosus. J Rheumatol 2008;35:2152-8.
8. Neville $C$, Clarke $A E$, Joseph $L$, et al. Learning from discordance in patient and physician global assessments of systemic lupus erythematosus disease activity. J Rheumatol 2000;27:675-9.

9. Yen JC, Abrahamowicz M, Dobkin PL, et al. Determinants of discordance between patients and physicians in their assessment of lupus disease activity. J Rheumatol 2003;30:1967-76.

10. Leong KP, Chong EY, Kong KO, et al. Discordant assessment of lupus activity between patients and their physicians: the Singapore experience. Lupus 2010;19:100-6.

11. Tamayo T, Fischer-Betz R, Beer S, et al. Factors influencing the health related quality of life in patients with systemic lupus erythematosus: long-term results (2001--2005) of patients in the German Lupus Erythematosus Self-Help Organization (LULA Study). Lupus 2010;19:1606-13.

12. Baker K, Pope J, Fortin P, et al. Work disability in systemic lupus erythematosus is prevalent and associated with socio-demographic and disease related factors. Lupus 2009;18:1281-8.

13. Yelin E, Tonner C, Trupin L, et al. Longitudinal study of the impact of incident organ manifestations and increased disease activity on work loss among persons with systemic lupus erythematosus. Arthritis Care Res (Hoboken) 2012;64:169-75.

14. Zink A, Listing J, Klindworth C, et al. The National Database of the German Collaborative Arthritis Centres: I. Structure, aims, and patients. Ann Rheum Dis 2001;60:199-206.

15. Lautenschlaeger J, Mau W, Kohlmann T, et al. [Comparative evaluation of a German version of the Health Assessment Questionnaire and the Hannover Functional Capacity Questionnaire] German. Z Rheumatol 1997;56:144-55.

16. Hart BI. Significance levels for the ratio of the mean square successive difference to the variance. Ann Math Statist 1942;13:445-7.

17. Belmont HM. Treatment of systemic lupus erythematosus-2013 update. Bull Hosp Jt Dis 2013;71:208-13.

18. van Vollenhoven RF, Mosca M, Bertsias G, et al. Treat-to-target in systemic lupus erythematosus: recommendations from an international task force. Ann Rheum Dis 2014;73:958-67.

19. Drenkard C, Bao G, Dennis G, et al. Burden of systemic lupus erythematosus on employment and work productivity: data from a large cohort in the southeastern United States. Arthritis Care Res (Hoboken) 2014;66:878-87.

20. Mau W, Thiele K, Lamprecht J. [Trends of work force participation of patients with rheumatic diseases : results from German social insurance data and the national database of the German collaborative arthritis centers]. Z Rheumatol 2014;73:11-19.

21. The information system of the Federal Health Monitoring. http://www. gbe-bund.de Diagnostic data of the hospitals. Average length of stay. Classification: years, age, sex, specialist department. 2014.

22. Huscher D, Mittendorf T, von HU, et al. Evolution of cost structures in rheumatoid arthritis over the past decade. Ann Rheum Dis Published Online First: 9 Jan 2014. doi:10.1136/annrheumdis-2013204311

23. Zink A, Fischer-Betz $\mathrm{R}$, Thiele $\mathrm{K}$, et al. Health care and burden of illness in systemic lupus erythematosus compared to rheumatoid arthritis: results from the National Database of the German Collaborative Arthritis Centres. Lupus 2004;13:529-36.

24. Mosca M, Bombardieri S. Assessing remission in systemic lupus erythematosus. Clin Exp Rheumatol 2006;24(6 Suppl 43) S-99-104

25. Huscher D, Albrecht K, Bischoff S, et al. Increasing disparities between physician assessment of disease acitivity and patient global health in Germany between 2000 and 2012. EULAR, Paris, 2014. Ann Rheum Dis 2014;73(supp 2):336 [abstract]. 